OPEN ACCESS

Edited by:

Joanna Trylska

University of Warsaw, Poland

Reviewed by:

Nanjie Deng,

Pace University, United States

Peter M. Kekenes-Huskey,

University of Kentucky, United States

Sanjib Senapati,

Indian Institute of Technology

Madras, India

*Correspondence:

Chia-en A. Chang

chiaenc@ucr.edu

Specialty section

This article was submitted to Biological Modeling and Simulation, a section of the journal

Frontiers in Molecular Biosciences

Received: 28 January 2021 Accepted: 06 April 2021 Published: 10 May 2021

Citation:

Kaushik S and Chang C-eA (2021) Molecular Mechanics Study of Flow and Surface Influence in Ligand-Protein Association.

Front. Mol. Biosci. 8:659687. doi: 10.3389/fmolb.2021.659687

\section{Molecular Mechanics Study of Flow and Surface Influence in Ligand-Protein Association}

\author{
Shivansh Kaushik and Chia-en A. Chang * \\ Department of Chemistry, University of Chemistry, Riverside, CA, United States
}

Ligand-protein association is the first and critical step for many biological and chemical processes. This study investigated the molecular association processes under different environments. In biology, cells have different compartments where ligand-protein binding may occur on a membrane. In experiments involving ligand-protein binding, such as the surface plasmon resonance and continuous flow biosynthesis, a substrate flow and surface are required in experimental settings. As compared with a simple binding condition, which includes only the ligand, protein, and solvent, the association rate and processes may be affected by additional ligand transporting forces and other intermolecular interactions between the ligand and environmental objects. We evaluated these environmental factors by using a ligand xk263 binding to HIV protease (HIVp) with atomistic details. Using Brownian dynamics simulations, we modeled xk263 and HIVp association time and probability when a system has xk263 diffusion flux and a non-polar self-assembled monolayer surface. We also examined different protein orientations and accessible surfaces for xk263. To allow xk263 to access to the dimer interface of immobilized HIVp, we simulated the system by placing the protein $20 \AA$ above the surface because immobilizing HIVp on a surface prevented xk263 from contacting with the interface. The non-specific interactions increased the binding probability while the association time remained unchanged. When the xk263 diffusion flux increased, the effective xk263 concentration around HIVp, xk263-HIVp association time and binding probability decreased non-linearly regardless of interacting with the self-assembled monolayer surface or not. The work sheds light on the effects of the solvent flow and surface environment on ligand-protein associations and provides a perspective on experimental design.

Keywords: molecular modeling, molecular recognition, drug design, GeomBD, ligand-receptor binding

\section{INTRODUCTION}

Molecular association is the first critical step in all chemical and biological processes such as the immune response, signal transduction, drug-protein binding, and chemical catalysis (Ozbabacan et al., 2010; Dill and Bromberg, 2012; Baron and McCammon, 2013; Lin et al., 2020). Simulation techniques play a crucial role in investigating the environment that may affect ligand-protein binding, which provides a fundamental understanding of molecular recognition and reduces the cost and time in molecular design. Although diffusion-controlled association rate constants may 
be approximated analytically, most ligand-protein systems have slower association rates than the diffusion-limited rate because the association event involves multiple steps (Di Cera, 2017; Pang and Zhou, 2017). Conformational rearrangement of both molecules largely determines their binding kinetics, but the two molecules must have an initial encounter first. This first step may be greatly affected by the environment, which can result in different measured association-rate constants.

Although many ligand-protein bindings take place in a closed system without water flow such as in an experimental beaker or cell, ligand-protein association can also occur in a more dynamic environment. Different compartments within a cell also create various membrane environments when ligands and proteins associate and function (Zotter et al., 2017). For example, techniques using continuous flow biocatalysis have been developed recently to improve the efficiency of chemical synthesis, such as improved mixing, mass transfer, and automation (Planchestainer et al., 2017; Britton et al., 2018). Surface plasmon resonance (SPR), a powerful technique to measure molecular binding kinetics and thermodynamics, also utilizes flow chemistry and a continuous flow environment (Hinman et al., 2018; Prabowo et al., 2018; Ershov et al., 2020). However, how the flow may affect the ligand-protein association processes is unclear. Moreover, these methods need to immobilize one molecule on a surface that may have intermolecular interactions with the molecules to be tested. Therefore, the choice of surface and flow rate usually need to be optimized for various systems.

In addition to experimentally measured values such as association rate constants, molecular simulation can reveal atomistic details of molecular association to further interpret experimental results and understand binding mechanisms. Molecular encounter usually involves two molecules searching for each other in a vast space and for longer than a nanosecond search time. Brownian dynamics (BD) simulations have been used for computational assessment of the encounter processes for several decades (Northrup et al., 1984; Huber and McCammon, 2019). Many software packages are available for studying a variety of problems related to bimolecular association. For example, MacroDox, UHBD, BrownDye Simulation of Diffusional Association (SDA), BD_BOX, BROMOC, ReaDDy, Smoldyn, SEEKR, and BDpack are used to probe ligand-protein associations with a flexible or rigid biomolecular model (Madura et al., 1995; Northrup et al., 1999; Huber and McCammon, 2010; Długosz et al., 2011; Schöneberg and Noé, 2013; De Biase et al., 2015; Martinez et al., 2015; Saadat and Khomami, 2015; Andrews, 2017; Votapka et al., 2017). Our group has been developing the GeomBD program, which focuses on using BD to investigate inter-enzyme intermediate transfer and substrate association on surface environments in biosensor and nanoenzyme structures (Roberts and Chang, 2015, 2016). Because of the diverse biosystems and binding environments, modifying an existing BD package to answer various questions is common practice.

Here we used the HIV protease (HIVp) and xk263 as a model system to study the effect of flow and surface on ligand-protein associations. HIVp belongs to the class of aspartyl proteases and is essential for maturation and assembly of infectious virions
(Kohl et al., 1988). The protein cleaves the large polyprotein precursors to mature viral proteins (Huang et al., 2014), an essential function for viral replication, and is a major drug target for AIDS treatment. This is a well-studied system with rich experimental binding data for many inhibitors and FDA approved drugs (Ghosh et al., 2016). The flexible flaps of HIVp can serve as a gate: its opening and closing affects ligand binding. In proteins, an open/closed rate of a gate related to the diffusion of their binding partners determines a fast or slow gating for ligand binding (Szabo et al., 1982; McCammon, 2011). Because of the complex gating behavior of HIVp and its importance in therapeutics, earlier work applied BD to study drug-HIVp binding and also developed a specialized coarse-grained model for HIVp to model the large-scale motions of the flaps (Tozzini and McCammon, 2005; Chang et al., 2006; Kang et al., 2011; Li et al., 2012; Bernetti et al., 2019).

In this work, we used BD simulations to examine the xk263HIVp association under the influence of ligand diffusion flux and a surface environment. The simulation applied rigid-body $\mathrm{BD}$ movements and considered atomistic details using precomputed grids when computing intermolecular interactions and driving forces. The HIVp was immobilized on a surface or artificially placed $20 \AA$ above the surface. We introduced a self-assembled monolayer (SAM) with a $\mathrm{CH}_{3}$ terminal group to model a hydrophobic surface environment (Cholko et al., 2019). We analyzed ligand association time and binding probability with different diffusion fluxes of xk263, surface and HIVp orientations. The non-polar SAM provided only weak intermolecular attractions with xk263, but the surface did not accelerate the $\mathrm{xk} 263$ encounter processes. The $\mathrm{x}$-direction diffusion flux of xk263 significantly reduced weak intermolecular interactions and searching near the surface which shortened the association time but also significantly reduced the probability of successful binding. The concentration gradient of xk263 was affected by the xk263 diffusion flux as well. Our studies suggest that the flow may have noticeable effects on molecular encounter processes and provide insights into the differences in the ligandprotein association in diverse conditions, such as in static cells or a continuous flow biocatalysis environment.

\section{METHODS}

\section{Model System}

The model system is a rectangular prism $(400 \times 400 \times 220$ $\AA^{3}$ ), closed at the top, and consisting of HIVp, xk263, and a SAM surface (Figure 1A). The crystal structure of HIVp and the xk263 inhibitor were obtained from the Protein Data Bank (codes 1HHP and 1HVR, respectively) (Spinelli et al., 1991; Lam et al., 1994). The HIVp flaps, two polypeptides that cover the active site, are in the open position. The semi-open form crystal structure of HIVp was simulated to derive the open form (Supplementary Methods) (Huang et al., 2017). HIVp was kept fixed in the model (Figure 1B). The SAM surface with HIVp at the center consisted of undecanethiol chains on a gold sheet of 1 atom thickness (Supplementary Methods) (Cholko et al., 2019). The size of the SAM was $400 \times 400 \AA^{2}$ having a hexagonal packing pattern with a packing density in the order of $10^{14} \mathrm{~cm}^{-2}$, which 
A
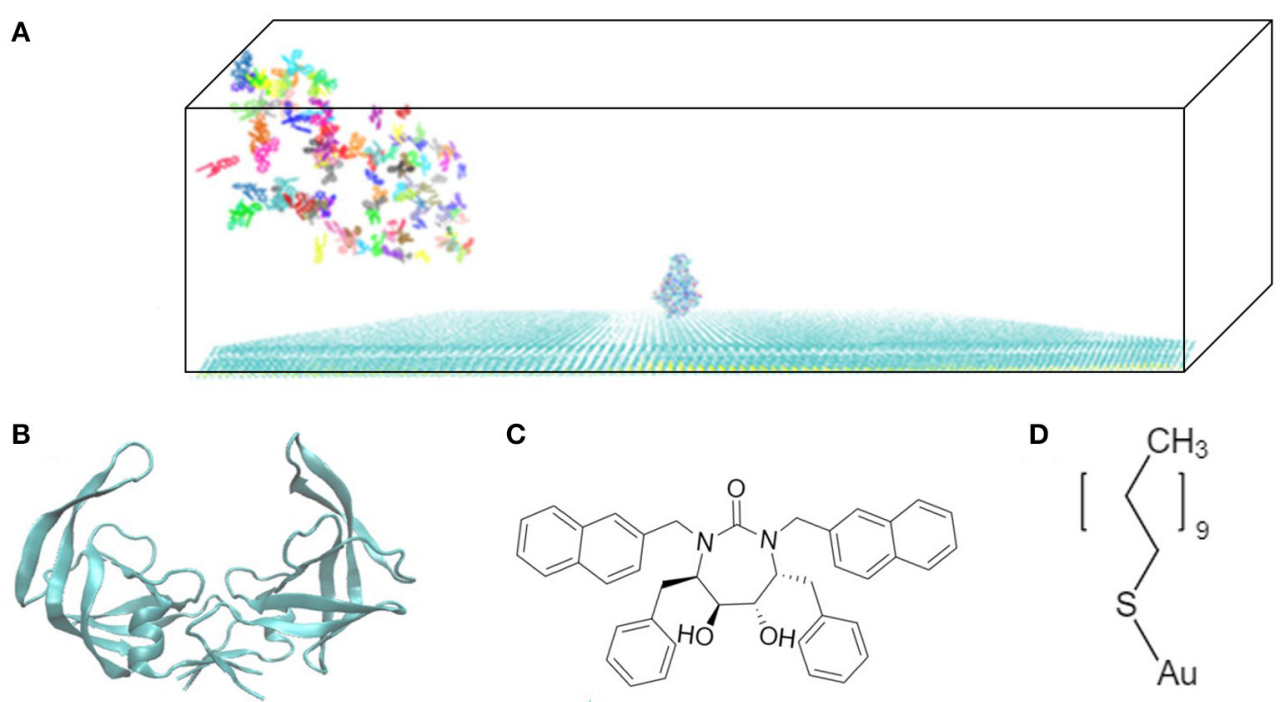

C<smiles>O=C1N(Cc2ccccc2)[C@@H](Cc2ccccc2)[C@@H](O)[C@H](O)[C@H](Cc2ccccc2)N1Cc1ccc2ccccc2c1</smiles>

D

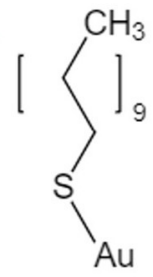

FIGURE 1 | Model system. (A) HIVp is placed at the center of the $\mathrm{CH}_{3}$-SAM surface with xk263 ligands in a yz-plane when a simulation starts. (B) Structure of an open-form HIVp in cartoon representation. (C) xk263 ligand, and (D) undecanethiol molecule used to build the SAM surface. Notably, in each BD run, the molecular system has only one xk263 and one HIVp. To speed up the calculations, multiple replicas of xk263 are simulated simultaneously, but the replicas do not interact with each other.

\section{X-direction}
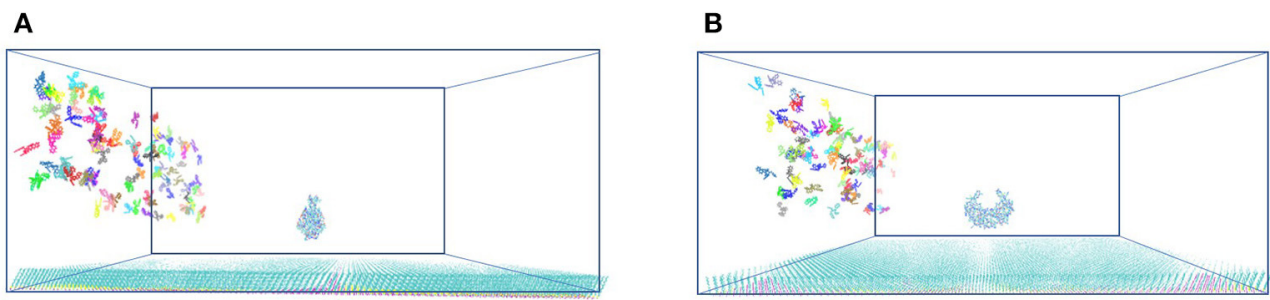

FIGURE 2 | Two orientations of HIVp. The protein is placed $20 \AA$ above the SAM (A) parallel to the yz-plane and (B) perpendicular to the yz-plane.

gives an average chain separation of $4.98 \AA$. The system had a periodicity in the $y$-direction and termination boundary in the $+\mathrm{x}$-direction as the ligand flows in the $+\mathrm{x}$-direction. HIVp had two orientations (Figure 2).

\section{Simulation Details}

The in-house modified GeomBD2 program was used for all BD runs (Roberts and Chang, 2016). The program first creates three grid files: the exclusion volume, screened Coulumbic potential, and 12-6 Lennard-Jones potential for HIV and SAM. Intermolecular interactions between ligand xk263 and HIVp or SAM are grid-based calculations, and the forces are estimated numerically (Roberts and Chang, 2016). To speed up the calculations, these precalculated grids are extended up to 40 and $15 \AA$ around HIVp or SAM for screened Coulumbic and 12-6 LJ potentials, respectively. The force field parameters were obtained from Amber-ff14SB for HIVp and $\mathrm{CH}_{3}$-SAM. The partial charges of xk263 and SAM were computed by using the AM1-BCC charge method with the antechamber program (Cholko et al., 2019). The motion of the ligand is a Brownian motion, governed by an overdamped Langevin equation, in implicit water (Northrup et al., 1984).

$$
r_{i}(t+\Delta t)=r_{i}(t)-\frac{D_{i}}{k_{B} T} \frac{\partial E}{\partial r_{i}} \Delta t+\sqrt{2 D_{i} \Delta t} R
$$

where $\mathrm{D}_{\mathrm{i}}$ is the translational or rotational diffusion coefficient, $\mathrm{k}_{\mathrm{B}}$ is Boltzmann's constant, $\mathrm{T}$ is temperature, $\delta \mathrm{E} / \delta \mathrm{r}_{\mathrm{i}}$ is the potential gradient computed numerically based on the potential grids, $\Delta \mathrm{t}$ is the time step, and $\mathrm{R}$ is the stationary Gaussian random number with a zero mean. To obtain the flow for the ligand, the overdamped Langevin equation is modified by adding $N$, a small fractional number, in the displacement of the $\mathrm{x}$-direction.

$$
x_{i}(t+\Delta t)=x_{i}(t)-\frac{D_{i}}{k_{B} T} \frac{\partial E}{\partial x_{i}} \Delta t+\sqrt{2 D_{i} \Delta t} R+N
$$


For each molecular system, we terminated the simulation when the runs generated at least $600 \times k 263-\mathrm{HIVp}$ associates and the computed average association time was within the standard deviation of $<3 \%$. A successful $x k 263-H I V p$ association is defined as xk263 reaches within $11.5 \AA$ ASP25, a residue in the active site of HIVp, and the association time is recorded. Notably, after generating 600 successful bindings, the computed average associating time for each system is within $3 \%$.

In the system, xk263 starts diffusing from the yz-plane at the - $\mathrm{x}$-direction boundary (Figure 1A). A run is ended when xk263 reaches the binding pocket or the $+\mathrm{x}$-direction boundary, and another new run will be started by randomly placing xk263 in a new position on the yz-plane (Figure 3). The clock random number seed is used, so no two runs can be identical. In addition, we can save trajectories of our $\mathrm{BD}$ runs to visualize ligand diffusion and binding/unbinding events.

\section{Calculations for System Analysis}

For the calculations of diffusion flux, diffusion coefficients, $\mathrm{CH}_{3}$ SAM interaction, and $\mathrm{xk} 263$ distribution, we used the trajectories with no termination at the $+\mathrm{x}$-direction boundary. So, the trajectories used for the calculations are continuous and $0.3-\mu \mathrm{s}$ long for each case.

\section{xk263 Diffusion Flux}

The diffusion flux is the rate of molecules transferred across the plane per unit time per unit area, representing the flow of xk263. It was calculated by using the continuous $\mathrm{BD}$ trajectories and reported in units of molecules $/ \mathrm{s} . \mathrm{m}^{2}$. The plane is an imaginary plane at the $+\mathrm{x}$ - directional boundary perpendicular to the direction of flow. The calculated flux densities according to the $\mathrm{N}$ values (in Equation 2) are reported (Supplementary Table 1).

\section{Diffusion Coefficient}

The diffusion coefficient was calculated by Einstein's relation, $<r^{2}>=2 \mathrm{nDt}$, where $\mathrm{r}$ is the displacement in time $\mathrm{t}, \mathrm{n}$ is the dimensionality, and D is the diffusion coefficient.

\section{$\mathrm{CH}_{3}$-SAM-xk263 Interaction Energy}

To calculate the interaction energetics of xk263 with $\mathrm{CH}_{3}$ SAM, we used the selected portion of continuous trajectories with xk263 diffusions within $25 \AA$ above the $\mathrm{CH}_{3}$-SAM. The interaction energy includes 12-6 Lennard-Jones potential and screened Coulombic potential. The cutoffs for vdW and electrostatic potential were $12 \AA$ and $40 \AA$, respectively.

The calculation of 12-6 Lennard-Jones potential is as follows:

$$
E_{v d w}=4 \in\left(\frac{\sigma^{12}}{r_{i j}^{12}}-\frac{\sigma^{6}}{r_{i j}^{6}}\right)
$$

where $r_{i j}$ is the distance between atoms $i$ and $j, \epsilon$ is the pairwise well-depth parameter and $\sigma$ is the distance at which the atomic radii meet and where the potential changes sign.

$$
\begin{array}{r}
\sigma=\frac{\sigma_{i}+\sigma_{j}}{2} \\
\epsilon=\sqrt{\epsilon_{i} \epsilon_{j}}
\end{array}
$$

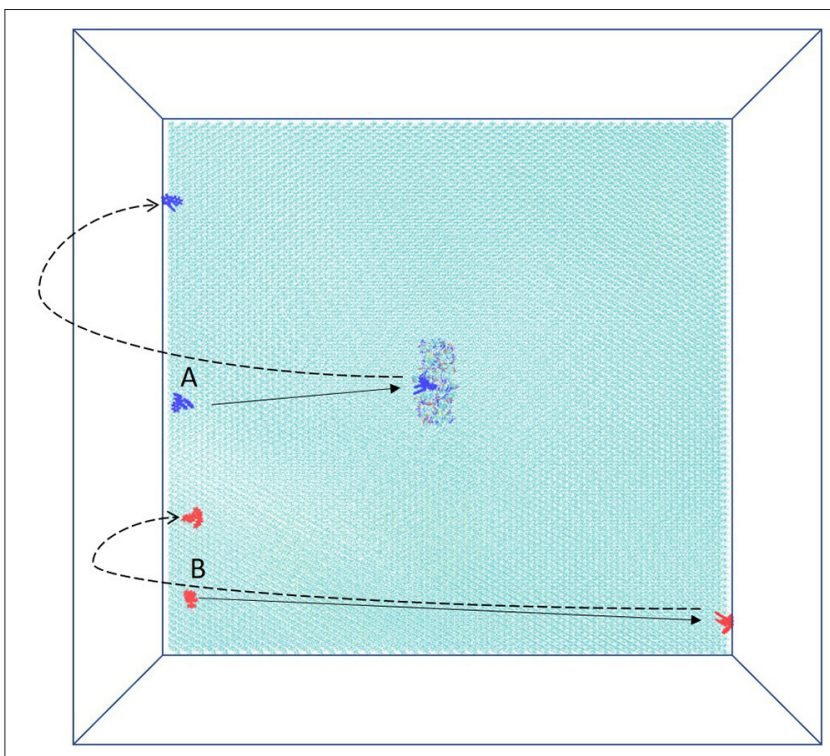

FIGURE 3 | Schematic top view of the simulation box. HIVp is placed on $\mathrm{CH}_{3}$-SAM, and two initial starting positions of $x \mathrm{k} 263, \mathrm{~A}$ and $\mathrm{B}$, are on the yz-plane. The simulation is terminated after xk263 binding to HIVp, and another new replica will start. If $x k 263$ reaches the $+x$-direction wall, the simulation is also terminated, and a new replica will start in a different position on the yz-plane.

TABLE 1 | Summary of molecular systems used in this study.

\section{Details of model system}

HIVp orientations 2

Periodic wall $y$

Flow direction $\quad x$

Top Closed in z-direction

Ligand concentration $\quad 110 \mu \mathrm{M}$

Ligand initial position As a yz-plane

Surface with and without $\mathrm{CH}_{3}$-SAM

Protein height $\quad 0$ and $20 \AA$ above the surface

Box dimension $\quad 400 \times 400 \times 220 \AA^{3}$

Termination Reaching $+x$-direction wall or within 11.5 Å of ASP $25^{\prime}$

The calculation of screened Coulombic potential is as follows:

$$
E_{S C}=\frac{k_{e} q_{i} q_{j} e^{-k r_{i j}}}{r_{i j} \epsilon}
$$

where $r_{i j}$ is the distance between two point-charges $i$ and $j$ from the ligand and receptor, respectively, $\mathrm{q}_{i}$ and $\mathrm{q}_{j}$ are the partial electron charges of the ligand point charges $i$ and receptor point charges $\mathrm{j}, \mathrm{k}$ is the screening parameter, $\mathrm{k}_{\mathrm{e}}$ is the Coulomb constant, $\mathrm{kb}$ is the Boltzmann constant and $\epsilon$ is the solution dielectric constant.

\section{xk263 Distribution}

The distribution of xk263 in the system was calculated by using continuous BD trajectories. The volume of the system was 
divided into 11 slices of $20 \AA$ height each. The total number of appearances of xk263 replicas was computed for each slice in $0.3 \mu \mathrm{s}$. The calculation of $g(z)$, distribution, is according to Equation 8

$$
g(z)=\frac{N}{M}=\frac{N}{n_{R} \times \frac{v}{V} \times f}
$$

where $\mathrm{N}$ is the total number of appearances of $\mathrm{xk} 263$ replicas in a slice within a certain number of frames, $M$ is the expected total number of appearances approximated from a random diffusional motion, $\mathrm{n}_{\mathrm{R}}$ is the number of $\mathrm{xk} 263$ replicas in the system, $\mathrm{v}$ is the volume of the slice, $\mathrm{V}$ is the total volume of the system, and $\mathrm{f}$ is the number of frames to be analyzed.

\section{RESULTS AND DISCUSSION}

Understanding ligand-receptor associations in various environments is of vital importance in drug binding in cells or in different experiments. In this study, we analyzed the effects of the SAM surface, the diffusion flux of ligand xk263 and the position of HIVp from the surface and in comparison with results with a static environment (Table 1). HIVp has flexible flaps, which are mostly in closed conformations (Katoh et al., 2003; Kang et al., 2011). To bind with a ligand, the flaps may open spontaneously or are induced to open by the ligand. Because this study focuses on the initial molecular encounter processes, we chose a flap open conformation and used rigid-body $\mathrm{BD}$ simulations to model the ligand association. In addition, the orientation of HIVp with respect to the $+\mathrm{x}$ direction diffusion flux of xk263 can affect ligand association. Therefore, we examined two HIVp orientations, perpendicular and parallel with the ligand flux (Figure 2). We modeled 60 different systems (Figure 4). We chose the values of xk263 diffusion flux that gradually show the changes of the modeled values with the flux.

\section{Environmental Factors Involved in Ligand Diffusion and Distribution Intermolecular Interactions Between xk263 and $\mathrm{CH}_{3}$-SAM}

We first examined the interactions between xk263 and $\mathrm{CH}_{3}$ SAM when the ligand diffusion flux varies. Table 2 shows weak van der Waals (vdW) interactions between xk263 and $\mathrm{CH}_{3}-\mathrm{SAM}$ regardless of the flux, which resulted in no significant adsorption of $x k 263$ on SAM (Figure 6A). Because of the hydrophobic nature of $\mathrm{CH}_{3}$-SAM and $\mathrm{xk} 263$, it is not surprising that the electrostatic interaction was close to zero.

Notably, our BD movement yielded the translational diffusion coefficient of xk263, $\mathrm{D}_{\text {lig }}=5.58 \pm 0.33 \times 10^{-6} \mathrm{~cm}^{2} / \mathrm{s}$ when a system had no SAM and no ligand diffusion flux. The modeled ligand diffusion coefficient is in good agreement with analytical values, $D_{\text {analytical }}=4.91 \times 10^{-6} \mathrm{~cm}^{2} / \mathrm{s}$, which also validates our simulation setting and BD algorithm (Supplementary Methods). Although the intermolecular vdW was weak, the attractions still affected the diffusion of xk263 molecules, and the molecule diffused a little slower in the presence of SAM with or without xk263 diffusion flux (Figure 5, Supplementary Table 1). Because the flow rate is inversely proportional to the viscosity (Pfitzner, 1976), the diffusion coefficient of xk263 increased with increasing

TABLE 2 | Interaction energies between xk263 and $\mathrm{CH}_{3}$-SAM in 7 different xk263 diffusion fluxes.

\begin{tabular}{lcc}
\hline $\mathbf{x k 2 6 3}$ diffusion flux $\left(\mathbf{~} \mathbf{1 0}^{\mathbf{2 2}} \mathbf{/ s . \mathbf { m } ^ { 2 } )}\right.$ & $\mathbf{E}_{\text {elec }}(\mathbf{k c a l} / \mathbf{m o l})$ & $\mathbf{E}_{\mathbf{v d w}}(\mathbf{k c a l} / \mathbf{m o l})$ \\
\hline 0 & $-0.045 \pm 0.14$ & $-1.126 \pm 0.89$ \\
0.45 & $-0.056 \pm 0.12$ & $-1.098 \pm 0.65$ \\
1.09 & $-0.010 \pm 0.11$ & $-1.547 \pm 1.14$ \\
3.43 & $0.015 \pm 0.14$ & $-1.194 \pm 0.95$ \\
5.62 & $0.003 \pm 0.11$ & $-1.488 \pm 0.96$ \\
11.29 & $0.006 \pm 0.10$ & $-1.273 \pm 1.13$ \\
17.20 & $0.005 \pm 0.13$ & $-1.069 \pm 0.83$
\end{tabular}

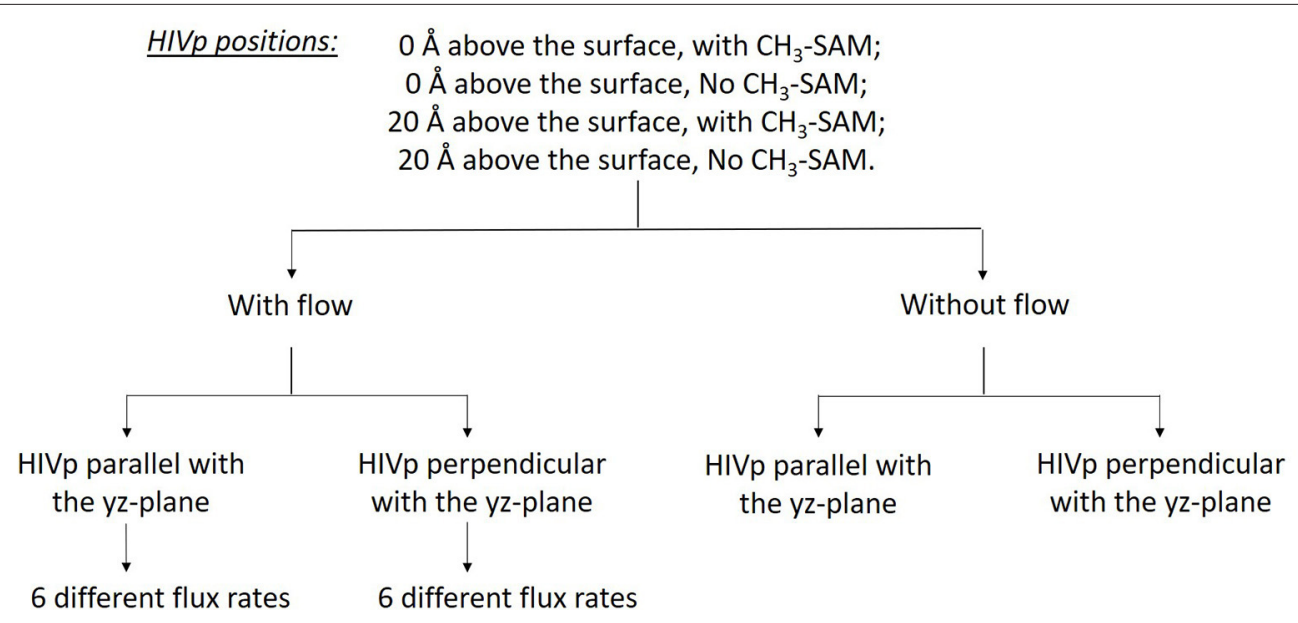

FIGURE $\mathbf{4}$ | Sixty different systems used in this study. The two HIVp orientations are shown in Figure 2. 
xk263 diffusion flux, as anticipated. However, because the water flow rate was not equal to the xk263 diffusion flux, we did not see a simple linear relationship between the two values.

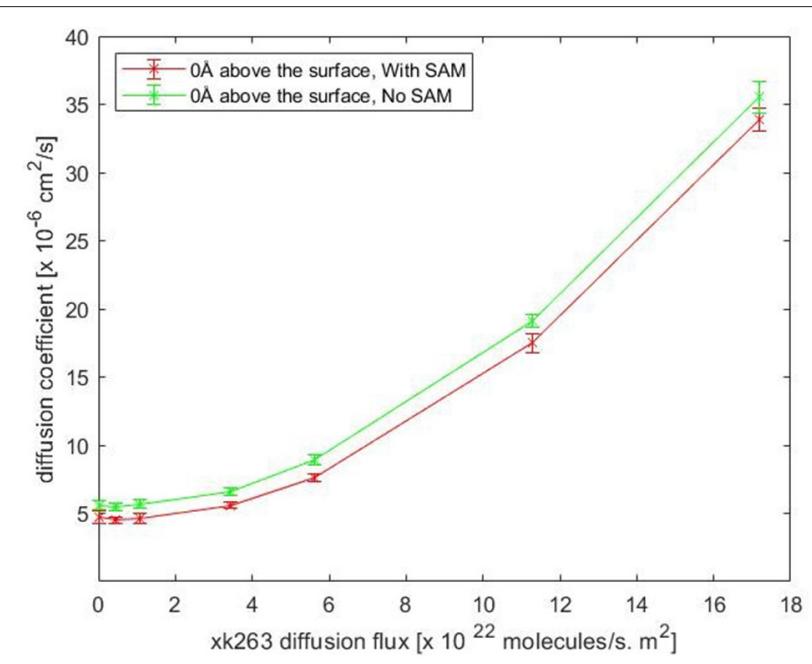

FIGURE 5 | Plot of translational diffusion coefficient of xk263. Diffusion coefficient increases with xk263 diffusion flux. (see Supplementary Table 1 for numerical data).

\section{Distribution of xk263 in the Simulation Tube}

We further investigated whether the environment may affect the concentration gradient of xk263 along the $\mathrm{z}$-direction when the molecule diffuses in the square tube. The system was partitioned into slices of $20 \AA$ each along z-direction, and Figure 6 shows the distribution of $x k 263$ as a function of their distance above the surface. The distribution of $x k 263$ for a slice, $\mathrm{g}(\mathrm{z})$, is the ratio of number of observed $\mathrm{xk} 263$ and number of expected xk263 approximated from a random diffusional motion. The systems include HIVp with one orientation shown in Figure 2A. When there was no SAM and the xk263 diffusion flux was small (green line in Figure 6A), the distribution of $\mathrm{xk} 263$ was the same throughout the $\mathrm{z}$-direction (distribution ratio $=\sim 1$ ). In contrast, when $\mathrm{CH}_{3}-\mathrm{SAM}$ was present (Figures 6A-C), xk263 was double that in the first slide (within $20 \AA$ of the SAM) when diffusion flux of xk263 was $<1.09 \times 10^{22}$ molecules/s $\mathrm{m}^{2}$. The results suggest that even if the intermolecular attraction between $\mathrm{xk} 263$ and $\mathrm{CH}_{3}-\mathrm{SAM}$ is weak, the concentration can be increased by 2 -fold, which helps to increase the probability of a molecular encounter. Studies for various systems also showed that local molecular concentration can be effected by a surface; for example, surface catalyzed biomolecular reaction using nanoreactors, nucleotidase colocalization, and absorption of biomacromolecules on hydrogels (Roa et al., 2017; Pérez-Mas et al., 2018; Rahmaninejad et al., 2020).

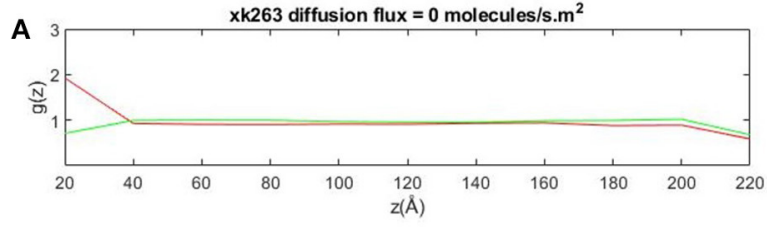

C

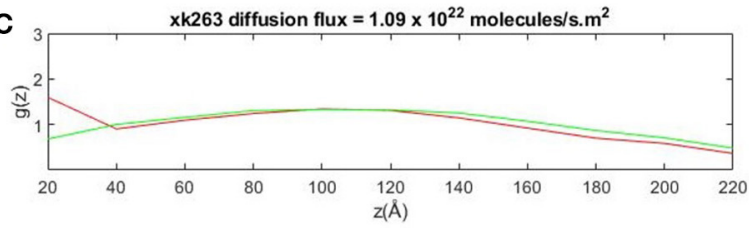

E
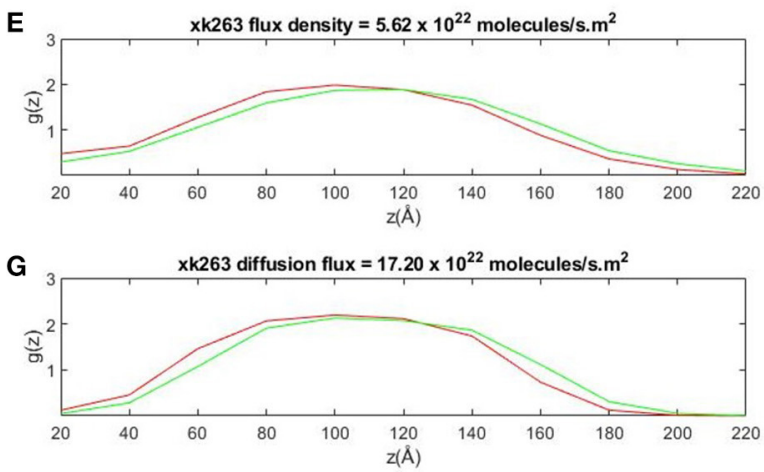

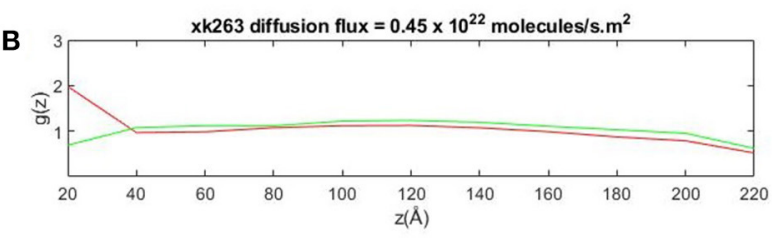

D

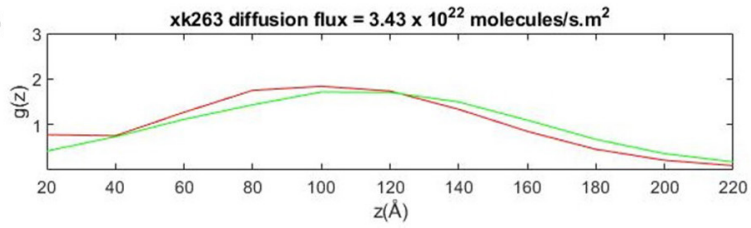

$\mathbf{F}$

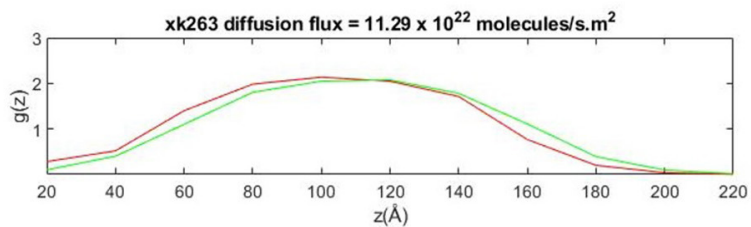

$O \AA$ above the surface, With SAM $D \AA$ above the surface, No SAM

FIGURE 6 | Plot of concentration distribution of xk263, $\mathrm{g}(\mathrm{z})$, as a function of the distance above the $\mathrm{CH}_{3}-\mathrm{SAM}$, $\mathrm{z}$, in seven different diffusion fluxes (A-G). The space was partitioned into equally sized slices with a height of $20 \AA . \mathrm{g}(\mathrm{z})$ is the ratio of number of observed xk263 and the number of expected xk263 approximated from a random diffusional motion. 
TABLE 3 | Average association time ( $\mathrm{t}_{\text {avg }}$ ) with different xk263 diffusion fluxes.

\begin{tabular}{|c|c|c|c|c|c|c|c|c|}
\hline \multirow[b]{2}{*}{$\begin{array}{l}\text { xk263 diffusion } \\
\text { flux }\left(\times 10^{22}\right. \\
\left./ \mathrm{s} . \mathrm{m}^{2}\right)\end{array}$} & \multicolumn{4}{|c|}{ HIVp perpendicular to the $y z-p l a n e, t_{\text {avg }}$ (ns) } & \multicolumn{4}{|c|}{ HIVp parallel to the yz-plane, $t_{\text {avg }}$ (ns) } \\
\hline & $\begin{array}{c}\text { 0A above the } \\
\text { surface, With } \\
\text { SAM }\end{array}$ & $\begin{array}{c}\text { 0A above the } \\
\text { surface, No } \\
\text { SAM }\end{array}$ & $\begin{array}{c}20 \AA \text { above the } \\
\text { surface, With } \\
\text { SAM }\end{array}$ & $\begin{array}{c}20 \AA \text { above the } \\
\text { surface, No } \\
\text { SAM }\end{array}$ & $\begin{array}{c}\text { OA above the } \\
\text { surface, With } \\
\text { SAM }\end{array}$ & $\begin{array}{c}\text { 0A above the } \\
\text { surface, No } \\
\text { SAM }\end{array}$ & $\begin{array}{c}20 \AA \text { above the } \\
\text { surface, With } \\
\text { SAM }\end{array}$ & $\begin{array}{c}20 \AA \text { above the } \\
\text { surface, No } \\
\text { SAM }\end{array}$ \\
\hline 0 & 1491.55 & 1064.41 & 1213.45 & 901.99 & 1405.77 & 1084.49 & 1124.5 & 905.21 \\
\hline 0.45 & 126.50 & 122.00 & 108.70 & 110.17 & 145.54 & 124.90 & 128.30 & 111.68 \\
\hline 1.09 & 55.56 & 51.93 & 43.61 & 45.28 & 53.71 & 50.05 & 47.98 & 44.65 \\
\hline 3.43 & 18.73 & 18.83 & 15.042 & 15.57 & 17.77 & 17.83 & 16.23 & 15.53 \\
\hline 5.62 & 11.08 & 10.48 & 10.31 & 9.47 & 10.78 & 11.01 & 9.75 & 9.55 \\
\hline 11.29 & 5.62 & 5.52 & 5.52 & 4.80 & 5.53 & 5.38 & 4.89 & 4.89 \\
\hline 17.20 & 3.69 & 3.59 & 3.33 & 3.25 & 3.58 & 3.69 & 3.27 & 3.26 \\
\hline
\end{tabular}

TABLE 4 | Inverse of average association time per molarity and percentage of successful binding with different xk263 diffusion fluxes.

\begin{tabular}{|c|c|c|c|c|c|c|c|c|}
\hline \multirow{2}{*}{$\begin{array}{l}\text { xk263 diffusion } \\
\text { flux }\left(x 10^{22}\right. \\
\left./ s . m^{2}\right)\end{array}$} & \multicolumn{2}{|c|}{$\begin{array}{c}\text { 0A above the surface, } \\
\text { With SAM }\end{array}$} & \multicolumn{2}{|c|}{$\begin{array}{c}\text { 0A above the surface, No } \\
\text { SAM }\end{array}$} & \multicolumn{2}{|c|}{$\begin{array}{c}20 \AA \text { above the surface, } \\
\text { With SAM }\end{array}$} & \multicolumn{2}{|c|}{$\begin{array}{c}20 \AA \text { above the surface, } \\
\text { No SAM }\end{array}$} \\
\hline & $\begin{array}{l}1 / t_{\text {avg }} \text { perM } \\
\text { (/nsM) }\end{array}$ & $\begin{array}{l}\text { Association } \\
\text { percentage }\end{array}$ & $\begin{array}{l}1 / t_{\text {avg }} \text { per } M \\
\text { (/nsM) }\end{array}$ & $\begin{array}{l}\text { Association } \\
\text { percentage }\end{array}$ & $\begin{array}{c}1 / t_{\text {avg }} \text { per } M \\
\text { (/nsM) }\end{array}$ & $\begin{array}{l}\text { Association } \\
\text { percentage }\end{array}$ & $\begin{array}{c}1 / t_{\text {avg }} \text { per } M \\
\text { (/nsM) }\end{array}$ & $\begin{array}{l}\text { Association } \\
\text { percentage }\end{array}$ \\
\hline \multicolumn{9}{|c|}{ HIVp perpendicular to the yz-plane } \\
\hline 0 & 6.09 & 18.59 & 8.54 & 18.14 & 7.49 & 18.67 & 10.08 & 19.18 \\
\hline 0.45 & 71.86 & 3.02 & 74.52 & 3.82 & 83.63 & 3.53 & 82.52 & 4.24 \\
\hline 1.09 & 163.63 & 1.22 & 175.07 & 1.73 & 208.45 & 1.67 & 200.75 & 2.16 \\
\hline 3.43 & 485.44 & 0.52 & 482.66 & 0.64 & 604.35 & 1.00 & 583.76 & 0.89 \\
\hline 5.62 & 820.41 & 0.35 & 867.45 & 0.33 & 881.41 & 0.90 & 959.31 & 0.65 \\
\hline 11.29 & 1618.17 & 0.18 & 1646.61 & 0.14 & 1646.43 & 0.70 & 1892.99 & 0.43 \\
\hline 17.20 & 2461.66 & 0.11 & 2532.29 & 0.07 & 2732.55 & 0.46 & 2793.33 & 0.34 \\
\hline \multicolumn{9}{|c|}{ HIVp parallel to the yz-plane } \\
\hline 0 & 6.47 & 17.15 & 8.38 & 17.85 & 8.08 & 18.99 & 10.04 & 20.61 \\
\hline 0.45 & 62.46 & 3.25 & 72.78 & 3.88 & 70.86 & 3.43 & 81.40 & 4.22 \\
\hline 1.09 & 169.25 & 1.21 & 181.64 & 1.71 & 189.48 & 1.86 & 203.61 & 1.92 \\
\hline 3.43 & 511.39 & 0.38 & 509.78 & 0.48 & 559.99 & 0.71 & 585.19 & 0.75 \\
\hline 5.62 & 842.87 & 0.22 & 825.62 & 0.21 & 931.88 & 0.40 & 952.29 & 0.42 \\
\hline 11.29 & 1645.34 & 0.05 & 1690.36 & 0.05 & 1858.74 & 0.14 & 1858.13 & 0.15 \\
\hline 17.20 & 2538.08 & 0.02 & 2461.19 & 0.02 & 2781.88 & 0.06 & 2788.36 & 0.07 \\
\hline
\end{tabular}

The SAM retains xk263 near the surface only when the diffusion flux of the ligand was small. When the flux increases, the local concentration of xk263 near the surface decreased, and xk263 had the highest concentration near the middle of the z-direction (see $\sim 100 \AA$ in Figures 6D-G). The distribution of concentration gradient along the $\mathrm{z}$-direction was the same with or without SAM, which suggests that the weak intermolecular interactions can be altered easily when xk263 has diffusion flux in the $\mathrm{x}$-direction. Our results are consistent with experiments showing the quadratic curve of concentration gradients with flow (Chen et al., 2017). With larger diffusion flux, a significantly smaller amount of xk263 diffused near the surface where HIVp locates, and the reduced distribution also contributed toward probability of small $\mathrm{xk} 263-\mathrm{HIVp}$ association, discussed later.

\section{HIVp-xk263 Association in Different Environments \\ HIVp Immobilized on the Surface}

We modeled the average xk263-HIVp association time and the binding percentage when HIVp was $0 \AA$ above the surface with SAM (Tables 3, 4 with SAM) or without SAM. Theoretically, if xk263 employs only 3 -dimensional diffusion within a sphere, the average association time to bind to HIVp located in the center of the sphere is $2648.4 \mathrm{~ns}$ when the system has no external flux (Supplementary Methods) (Adam and Delbrück, 1968). The association time modeled by our simulation is $1491.55 \mathrm{~ns}$ is close to the theoretical value. The faster binding time than theory is due to use of a box, and xk263 did not need to search the whole sphere to associate with HIVp. Notably, existing association rate theories describe system without diffusion flux (Berg and Purcell, 1977; Szabo et al., 1980; Shoup and Szabo, 

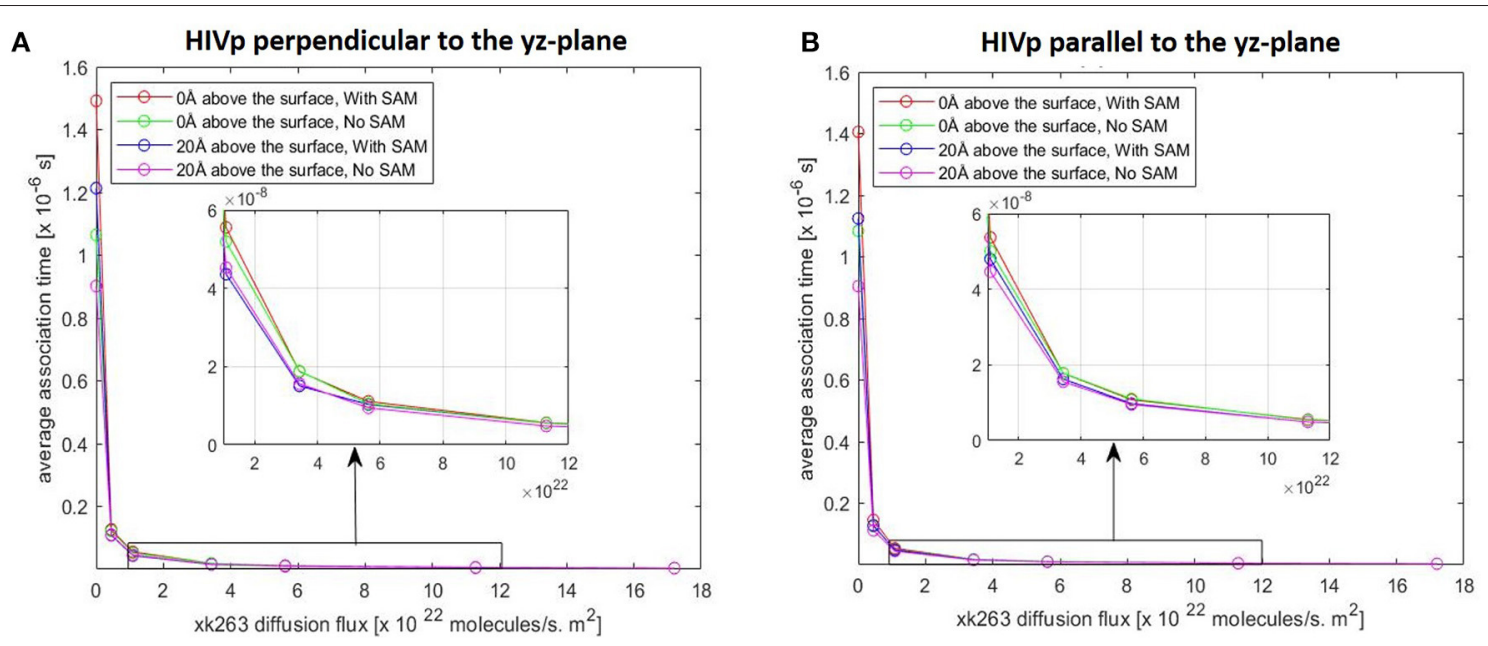

FIGURE 7 | Plot of average association time vs. xk263 diffusion flux. HIVp is placed (A) perpendicular to the yz-plane and (B) parallel to the yz-plane; Similar data imply no effect of HIVp orientation on the association time. In each plot, coinciding lines (red, green, blue, and magenta) show no effect of hydrophobic $\mathrm{CH}_{3}$-SAM or height of the receptor from the surface.
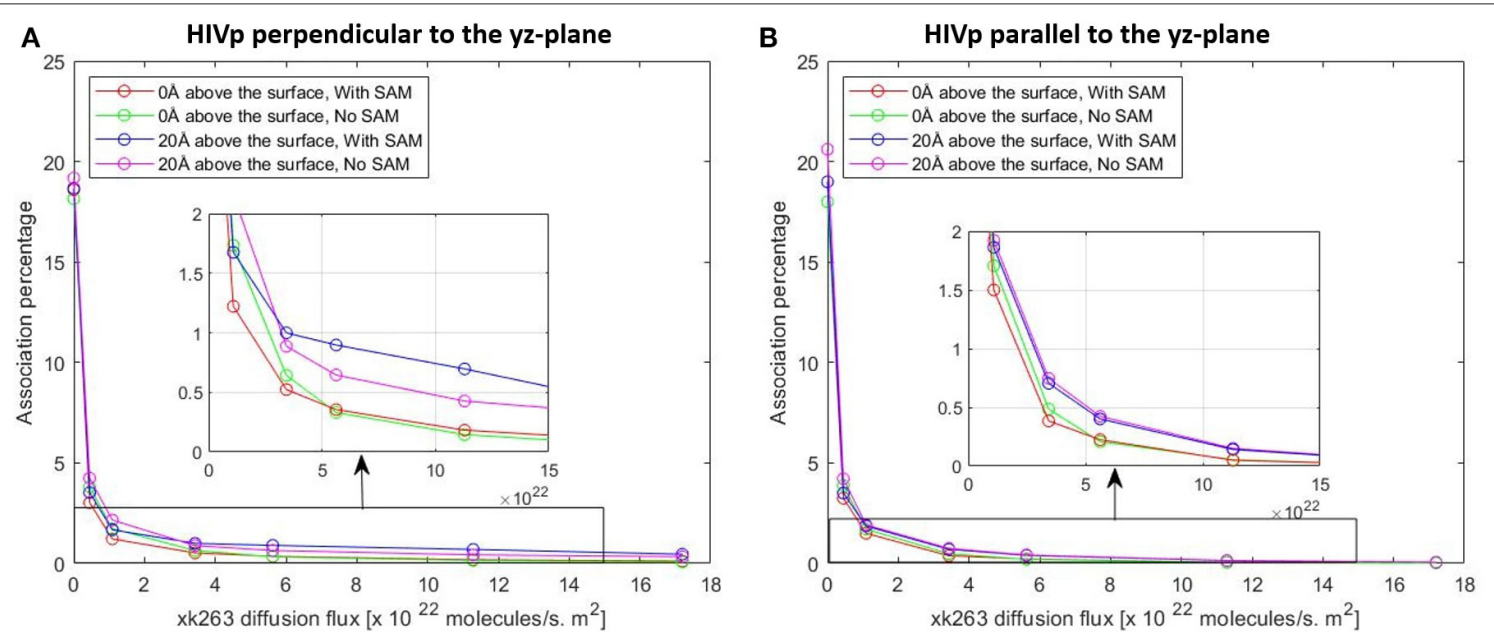

FIGURE 8 | Plot of association percentage vs. xk263 diffusion flux under two different HIVP's orientation (A,B). Association percentage is defined as the fraction of successful binding from the total number of trajectories in the system.

1982) and further theoretical work in molecular association with buffer flowing is needed. Although the interactions between xk263 and $\mathrm{CH}_{3}$-SAM were small, to examine the environment effects, we turned off the intermolecular vdW and electrostatic interactions, so the system was in a confined space without intermolecular attractions or repulsion (without SAM). The $\mathrm{x}$ direction diffusion flux of xk263 gradually increased from zero to $1.7 \times 10^{23}$ molecules/s $\mathrm{m}^{2}$, and the average $\mathrm{xk} 263-\mathrm{HIVp}$ association time decreased non-linearly. The correlation was observed in a previous study, showing that the association time for human immunoglobulin G (IgG) and Staphylococcus aureus protein A (SpA) decreased with increasing flow rate (Ogi et al., 2008). The average association time did not differ without or with SAM in the presence of diffusion flux (Table 3). This is not surprising because the $\mathrm{CH}_{3}-\mathrm{SAM}$ provides only weak attractions to $x k 263$, and the surface neither increased the local concentration of $x k 263$ to assist ligand association nor retained the ligand from binding to HIVp (Figure 6) (Cholko et al., 2020). However, if the attraction between a ligand and surface is large, it may affect experimental results. As a result, existing experimental studies considered the choice of the surface for the sensor or SPR to optimize experimental sensitivity and accuracy. For example, phospholipid/alkane bilayers might be a better option for molecular binding in biomolecular systems using SPR (Plant et al., 1995). These phospholipid derivatized surfaces may bring non-specific interactions for xk263 which is absent in our current model, which can result in slightly longer association time.

We report the inverse of association time per molarity and the association percentage (Table 4). When xk263 was freely diffused in the tube without diffusion flux, the association rate 
approximated using the average association time, 1/1491.55 ns per $\mathrm{M}$, yielded $\mathrm{k}_{\mathrm{on}}=7.8 \times 10^{9} 1 / \mathrm{Ms}$. Using the diffusion coefficient from the BD run (Supplementary Table 2), our simulated $\mathrm{k}_{\mathrm{on}}$ was approximately a half of the analytical value, $\mathrm{k}_{\mathrm{on}}=4 \pi \mathrm{RD}=1.2 \times 10^{10} 1 / \mathrm{Ms}$ because the analytical formula does not consider molecular geometry in association. Our modeled $\mathrm{k}_{\mathrm{on}}$ was also slightly slower than that measured using SPR for another highly similar cyclic urea compound, $2.5 \times 10^{10} 1 / \mathrm{Ms}$ (Markgren et al., 2002). The first phase in SPR utilizes buffer flowing and detects binding signals, which require successful collision to have the correct ligand and HIVp orientation and enough energy. The flow may influence successful collision, especially on biomolecular systems which usually have complex molecular geometry. Of note, the inverse association time increased linearly with xk263 diffusion flux (Supplementary Figure 1), which suggests that the association rate may also increase linearly with the flow. However, our modeled diffusion coefficients (Supplementary Table 2) show an exponential increase with the xk263 diffusion flux (Figure 5). When particles have flux moving along the $+\mathrm{x}$ direction, the ligand did not freely diffuse in all directions, and the equation for the diffusion-controlled association system, $\mathrm{k}_{\mathrm{on}}=4 \pi \mathrm{RD}$, was no longer suitable to approximate the association rate constants. Therefore, we cannot directly estimate $\mathrm{k}_{\mathrm{on}}$ from the increased diffusion coefficient.

The additional $+\mathrm{x}$ direction drift velocity also reduced the initial encounter probability. When the flux was $>3.43 \times 10^{22}$ molecules/s.m ${ }^{2}, x k 263$ passed the tube without sufficient time to freely diffuse in the $\mathrm{y}$ and $\mathrm{z}$ directions, which resulted in reduced search space and tremendously decreased association percentage. Large diffusion flux also yielded fewer xk263 molecules staying near both the SAM and HIVp surface. Notably, xk263 may diffuse on the surface of HIVp before binding to the pocket. The xk263 diffusion flux in the $+\mathrm{x}$ direction perturbed the interactions between xk263 and HIVp, and the weakened intermolecular attractions also led to decreased association percentage.

In typical experimental settings, an immobilized protein can have multiple orientations. Therefore, we chose two representative orientations-the binding pocket of HIVp perpendicular or parallel to the flux of xk263-to examine whether the orientation against the flow affects ligand binding. Tables 3, 4, Figures 7, 8 show no difference in average ligand association time and binding percentage with the two orientations. Because the binding pocket of HIVp is widely accessible for the ligand, the impact of the orientation was insignificant. However, some proteins exhibit a certain direction for ligands to enter the binding pocket, and their orientation against the flux of the ligands can affect the ligand-protein association.

\section{Artificially Localized HIVp $20 \AA$ Above the Surface}

Although different HIVp orientations did not affect xk263 association time, existing studies showed that the dimer interface (the bottom part of HIVp) is the most popular region for ligands' initial encounter of HIVp (Roberts and Chang, 2016).
After reaching the dimer interface, the ligand can undergo surface diffusion to reach the binding pocket. When we placed HIVp $0 \AA$ above the surface, this bottom region became partially inaccessible to xk263. Therefore, immobilized HIVp was artificially placed $20 \AA$ above the surface so that xk263 can easily reach the bottom region. When the whole HIVp surface was accessible to $\mathrm{xk} 263$, the association time was reduced by $10-20 \%$ when the $x k 263$ diffusion flux was $<1.09 \times 10^{22}$ molecules $/ \mathrm{s} . \mathrm{m}^{2}$, which suggests that the additionally available bottom region accelerates xk263 binding (Table 3) and slightly increases the association percentage (Table 4). Even when HIVp was placed $20 \AA$ above the surface, the location was not far enough to eliminate the intermolecular xk263-SAM interactions. As a result, xk263 spent longer time near SAM when xk263 diffusion flux was small, thus resulting in longer time to associate with HIVp when SAM was present rather than absent. When the flux exists, the association time was the same when the SAM was present or absent, regardless the position of HIVp (Table 3).

\section{CONCLUSION}

Ligand-protein encounters can occur in any environment that may provide additional intermolecular interactions or the transporting forces to the ligand. For example, in experimental settings such as using SPR to study ligand-protein binding, the choice of the surface and the flow rate are all optimized for measurements. In this study, we used BD simulations to investigate the effect of the $\mathrm{CH}_{3}-\mathrm{SAM}$ surface on xk263-HIVp association. The non-polar surface provided weak intermolecular $\mathrm{vdW}$ attractions with $\mathrm{xk} 263$ to slightly increase the ligand binding time. The effects quickly vanished when xk263 had an x-direction diffusion flux, and the association time decreased when the diffusion flux increased regardless of the presence of SAM or only a special plane. With no diffusion flux, xk263 was twice more concentrated within $20 \AA$ of the SAM surface because of the xk263-SAM interactions. The concentration gradient did not increase binding time but increased xk263-HIVp binding probability. When the xk263 diffusion flux increased, the middle region of the square tube had the highest xk263 concentration, which is the same as existing experiments showing a quadratic curve of concentration gradients with flow. The results also show that when HIVp was placed on the surface ( $0 \AA$ above the surface), the bottom part of the protein, a known high-probability site of the xk263-HIVp first encounter, was not accessible to xk263. Because xk263 could bind nonspecifically on the HIVp surface and then utilize surface diffusion to reach the binding site, occluding this bottom region increased ligand binding time by $10-20 \%$ in the static environment. However, with large xk263 diffusion flux, all the weak intermolecular interactions and searching along the HIVp surface were eliminated, which resulted in a fast association time and small binding probability. This work brings insights into how ligand diffusion flux and the environment may affect ligand-protein association. 


\section{DATA AVAILABILITY STATEMENT}

The raw data supporting the conclusions of this article will be made available by the authors, without undue reservation.

\section{AUTHOR CONTRIBUTIONS}

SK ran and analyzed the simulations, produced figures, and wrote the manuscript. $\mathrm{C}-\mathrm{eC}$ designed experiments, analyzed the simulation, and wrote the manuscript. Both authors contributed to the article and approved the submission version.

\section{FUNDING}

This study was supported by the US National Institutes of Health (GM-109045), US National Science Foundation

\section{REFERENCES}

Adam, G., and Delbrück, M. (1968). Reduction of dimensionality in biological diffusion processes. Struct. Chem. Mol. Biol. 198, 198-215.

Andrews, S. S. (2017). Smoldyn: particle-based simulation with rule-based modeling, improved molecular interaction and a library interface. Bioinformatics 33, 710-717. doi: 10.1093/bioinformatics/btw700

Baron, R., and McCammon, J. A. (2013). Molecular recognition and ligand association. Annu. Rev. Phys. Chem. 64, 151-175 doi: 10.1146/annurev-physchem-040412-110047

Berg, H. C., and Purcell, E. M. (1977). Physics of chemoreception. Biophys. J. 20, 193-219. doi: 10.1016/S0006-3495(77)85544-6

Bernetti, M., Masetti, M., Rocchia, W., and Cavalli, A. (2019). Kinetics of drug binding and residence time. Annu. Rev. Phys. Chem. 70, 143-171. doi: 10.1146/annurev-physchem-042018-052340

Britton, J., Majumdar, S., and Weiss, G. A. (2018). Continuous flow biocatalysis. Chem. Soc. Rev. 47, 5891-5918. doi: 10.1039/C7CS00906B

Chang, C.-E., Shen, T., Trylska, J., Tozzini, V., and McCammon, J. A. (2006). Gated binding of ligands to HIV-1 protease: brownian dynamics simulations in a coarse-grained model. Biophys. J. 90, 3880-3885. doi: 10.1529/biophysj.105.074575

Chen, X., Hu, Z., Zhang, L., Yao, Z., Chen, X., Zheng, Y., et al. (2017). Numerical and experimental study on a microfluidic concentration gradient generator for arbitrary approximate linear and quadratic concentration curve output. Int. J. Chem. Reactor Eng. 16. doi: 10.1515/ijcre-2016-0204

Cholko, T., Barnum, J., and Chang, C. E. (2020). Amyloid- $\beta$ (A $\beta 42)$ peptide aggregation rate and mechanism on surfaces with widely varied properties: insights from brownian dynamics simulations. J. Phys. Chem. B 124, 5549-5558. doi: 10.1021/acs.jpcb.0c02926

Cholko, T., Kaushik, S., and Chia-en, A. C. (2019). Dynamics and molecular interactions of single-stranded DNA in nucleic acid biosensors with varied surface properties. Phys. Chem. Chem. Phys. 21, 16367-16380. doi: 10.1039/C9CP02441G

De Biase, P. M., Markosyan, S., and Noskov, S. (2015). BROMOC suite: Monte Carlo/Brownian dynamics suite for studies of ion permeation and DNA transport in biological and artificial pores with effective potentials. J. Comput. Chem. 36, 264-271. doi: 10.1002/jcc.23799

Di Cera, E. (2017). Mechanisms of ligand binding. Biophys. Rev. 1:011303. doi: $10.1063 / 5.0020997$

Dill, K., and Bromberg, S. (2012). Molecular driving Forces: Statistical Thermodynamics in Biology, Chemistry, Physics, and Nanoscience. New York, NY: Garland Science. doi: 10.4324/9780203809075

Długosz, M., Zieliński, P., and Trylska, J. (2011). Brownian dynamics simulations on CPU and GPU with BD_BOX. J. Comput. Chem. 32, 2734-2744. doi: 10.1002/jcc.2 1847
(MCB-1932984), and NSF national supercomputer centers (TG-CHE130009).

\section{ACKNOWLEDGMENTS}

We thank Dr. Jason Quan Cheng for helpful discussions regarding SPR experiments. We thank Tim Cholko for help in modifying the GeomBD program and Jianan Sun for providing HIVp open structures.

\section{SUPPLEMENTARY MATERIAL}

The Supplementary Material for this article can be found online at: https://www.frontiersin.org/articles/10.3389/fmolb. 2021.659687/full\#supplementary-material
Ershov, P. V., Mezentsev, Y. V., Kaluzhskiy, L. A., and Ivanov, A. S. (2020). Phenanthridine derivatives as potential HIV-1 protease inhibitors. Biomed. Rep. 13:66. doi: 10.3892/br.2020.1373

Ghosh, A. K., Osswald, H. L., and Prato, G. (2016). Recent progress in the development of HIV-1 protease inhibitors for the treatment of HIV/AIDS. J. Med. Chem. 59, 5172-5208. doi: 10.1021/acs.jmedchem.5b01697

Hinman, S. S., McKeating, K. S., and Cheng, Q. (2018). Surface plasmon resonance: material and interface design for universal accessibility. Anal. Chem. 90:19. doi: 10.1021/acs.analchem.7b04251

Huang, X., Britto, M. D., Kear-Scott, J. L., Boone, C. D., Rocca, J. R., Simmerling, C., et al. (2014). The role of select subtype polymorphisms on HIV-1 protease conformational sampling and dynamics. J. Biol. Chem. 289, 17203-17214. doi: 10.1074/jbc.M114.571836

Huang, Y. M., Raymundo, M. A., Chen, W., and Chang, C. A. (2017). Mechanism of the association pathways for a pair of fast and slow binding ligands of HIV-1 protease. Biochemistry 56, 1311-1323. doi: 10.1021/acs.biochem.6b01112

Huber, G. A., and McCammon, J. A. (2010). Browndye: a software package for Brownian dynamics. Comput. Phys. Commun. 181, 1896-1905. doi: $10.1016 /$ j.cpc.2010.07.022

Huber, G. A., and McCammon, J. A. (2019). Brownian dynamics simulations of biological molecules. Trends Chem. 1, 727-738. doi: 10.1016/j.trechm.2019.07.008

Kang, M., Roberts, C., Cheng, Y., and Chang, C. E. (2011). Gating and intermolecular interactions in ligand-protein association: coarse-grained modeling of HIV-1 protease. J. Chem. Theory Comput. 7, 3438-3446. doi: $10.1021 /$ ct2004885

Katoh, E., Louis, J. M., Yamazaki, T., Gronenborn, A. M., Torchia, D. A., and Ishima, R. (2003). A solution NMR study of the binding kinetics and the internal dynamics of an HIV-1 protease-substrate complex. Protein Sci. 12, 1376-1385. doi: 10.1110/ps.0300703

Kohl, N. E., Emini, E. A., Schleif, W. A., Davis, L. J., Heimbach, J. C., Dixon, R., et al. (1988). Active human immunodeficiency virus protease is required for viral infectivity. Proce. Natl Acad. Sci. U.S.A. 85, 4686-4690. doi: $10.1073 /$ pnas.85.13.4686

Lam, P., Jadhav, P. K., Eyermann, C. J., Hodge, C. N., Ru, Y., Bacheler, L. T., et al. (1994). Rational design of potent, bioavailable, nonpeptide cyclic ureas as HIV protease inhibitors. Science 263, 380-384. doi: 10.1126/science.8278812

Li, D., Liu, M. S., Ji, B., Hwang, K. C., and Huang, Y. (2012). Identifying the molecular mechanics and binding dynamics characteristics of potent inhibitors to HIV-1 protease. Chem. Biol. Drug Design 80, 440-454. doi: 10.1111/j.1747-0285.2012.01417.x

Lin, Y.-C., Kim, W. K., and Dzubiella, J. (2020). Coverage fluctuations and correlations in nanoparticle-catalyzed diffusion-influenced bimolecular reactions. J. Phys. Chem. C 124, 24204-24214. doi: 10.1021/acs.jpcc.0c06898

Madura, J. D., Briggs, J. M., Wade, R. C., Davis, M. E., Luty, B. A., Ilin, A., et al. (1995). Electrostatics and diffusion of molecules in solution: simulations 
with the University of Houston Brownian Dynamics program. Comput. Phys. Commun. 91, 57-95. doi: 10.1016/0010-4655(95)00043-F

Markgren, P.-O., Schaal, W., Hämäläinen, M., Karlén, A., Hallberg, A., Samuelsson, B., et al. (2002). Relationships between structure and interaction kinetics for HIV-1 protease inhibitors. J. Med. Chem. 45, 5430-5439. doi: $10.1021 / \mathrm{jm} 0208370$

Martinez, M., Bruce, N. J., Romanowska, J., Kokh, D. B., Ozboyaci, M., Yu, X., et al. (2015). SDA 7: A modular and parallel implementation of the simulation of diffusional association software. J. Comput. Chem. 36, 1631-1645. doi: $10.1002 /$ jcc. 23971

McCammon, J. A. (2011). Gated diffusion-controlled reactions. BMC Biophys. 4, 1-5. doi: 10.1186/2046-1682-4-4

Northrup, S., Laughner, T., and Stevenson, G. (1999). MacroDox Macromolecular Simulation Program. Cookeville, TN: Tennessee Technological University, Department of Chemistry.

Northrup, S. H., Allison, S. A., and McCammon, J. A. (1984). Brownian dynamics simulation of diffusion-influenced bimolecular reactions. J. Chem. Phys. 80, 1517-1524. doi: 10.1063/1.446900

Ogi, H., Fukunishi, Y., Omori, T., Hatanaka, K., Hirao, M., and Nishiyama, M. (2008). Effects of flow rate on sensitivity and affinity in flow injection biosensor systems studied by $55-\mathrm{MHz}$ wireless quartz crystal microbalance. Anal. Chem. 80, 5494-5500. doi: $10.1021 / \mathrm{ac} 800459 \mathrm{~g}$

Ozbabacan, S. A., Gursoy, A., Keskin, O., and Nussinov, R. (2010). Conformational ensembles, signal transduction and residue hot spots: application to drug discovery. Curr. Opin. Drug Discov. Devel. 13, 527-537.

Pang, X., and Zhou, H.-X. (2017). Rate constants and mechanisms of protein-ligand binding. Ann. Rev. Biophys. 46, 105-130. doi: 10.1146/annurev-biophys-070816-033639

Pérez-Mas, L., Martín-Molina, A., Quesada-Pérez, M., and Moncho-Jordá A. (2018). Maximizing the absorption of small cosolutes inside neutral hydrogels: steric exclusion versus hydrophobic adhesion. Phys. Chem. Chem. Phys. 20, 2814-2825. doi: 10.1039/C7CP07679G

Pfitzner, J. (1976). Poiseuille and his law. Anaesthesia 31, 273-275. doi: 10.1111/j.1365-2044.1976.tb11804.x

Planchestainer, M., Contente, M. L., Cassidy, J., Molinari, F., Tamborini, L., and Paradisi, F. (2017). Continuous flow biocatalysis: production and in-line purification of amines by immobilised transaminase from Halomonas elongata. Green Chem. 19, 372-375. doi: 10.1039/C6GC01780K

Plant, A. L., Brighamburke, M., Petrella, E. C., and Oshannessy, D. J. (1995). Phospholipid/alkanethiol bilayers for cell-surface receptor studies by surface plasmon resonance. Anal. Biochem. 226, 342-348. doi: 10.1006/abio.1995.1234

Prabowo, B. A., Purwidyantri, A., and Liu, K.-C. (2018). Surface plasmon resonance optical sensor: a review on light source technology. Biosensors 8:80. doi: 10.3390/bios 8030080

Rahmaninejad, H., Pace, T., Bhatt, S., Sun, B., and Kekenes-Huskey, P. (2020). Colocalization and confinement of ecto-nucleotidases modulate extracellular adenosine nucleotide distributions. PLoS Comput. Biol. 16:e1007903. doi: 10.1371/journal.pcbi.1007903

Roa, R., Kim, W. K., Kanduc M., Dzubiella, J., and Angioletti-Uberti, S. (2017). Catalyzed bimolecular reactions in responsive nanoreactors. ACS Catal. 7, 5604-5611. doi: 10.1021/acscatal.7b01701
Roberts, C. C., and Chang, C.-E. (2016). Analysis of ligand-receptor association and intermediate transfer rates in multienzyme nanostructures with all-atom brownian dynamics simulations. J. Phys. Chem. B 120, 8518-8531 doi: 10.1021/acs.jpcb.6b0 2236

Roberts, C. C., and Chang, C. E. (2015). Modeling of enhanced catalysis in multienzyme nanostructures: effect of molecular scaffolds, spatial organization, and concentration. J. Chem. Theory Comput. 11, 286-292 doi: $10.1021 /$ ct5007482

Saadat, A., and Khomami, B. (2015). Matrix-free Brownian dynamics simulation technique for semidilute polymeric solutions. Phys. Rev. E 92:033307 doi: 10.1103/PhysRevE.92.033307

Schöneberg, J., and Noé, F. (2013). ReaDDy-a software for particle-based reaction-diffusion dynamics in crowded cellular environments. PLOS ONE 8:e74261(e2013). doi: 10.1371/journal.pone.0074261

Shoup, D., and Szabo, A. (1982). Role of diffusion in ligand binding to macromolecules and cell-bound receptors. Biophys. J. 40, 33-39. doi: 10.1016/S0006-3495(82)84455-X

Spinelli, S., Liu, Q., Alzari, P., Hirel, P., and Poljak, R. (1991). The three-dimensional structure of the aspartyl protease from the HIV1 isolate BRU. Biochimie 73, 1391-1396. doi: 10.1016/0300-9084(91) 90169-2

Szabo, A., Schulten, K., and Schulten, Z. (1980). First passage time approach to diffusion controlled reactions. J. Chem. Phys. 72, 4350-4357. doi: $10.1063 / 1.439715$

Szabo, A., Shoup, D., Northrup, S. H., and McCammon, J. A. (1982). Stochastically gated diffusion-influenced reactions. J. Chem. Phys. 77, 4484-4493. doi: 10.1063/1.444397

Tozzini, V., and McCammon, J. A. (2005). A coarse grained model for the dynamics of flap opening in HIV-1 protease. Chem. Phys. Lett. 413, 123-128. doi: 10.1016/j.cplett.2005.07.075

Votapka, L. W., Jagger, B. R., Heyneman, A. L., and Amaro, E. R. (2017). SEEKR: simulation enabled estimation of kinetic rates, a computational tool to estimate molecular kinetics and its application to trypsinbenzamidine binding. J. Phys. Chem. B 121, 3597-3606. doi: 10.1021/acs.jpcb. $6 \mathrm{~b} 09388$

Zotter, A., Bäuerle, F., Dey, D., Kiss, V., and Schreiber, G. (2017). Quantifying enzyme activity in living cells. J. Biol. Chem. 292, 15838-15848. doi: 10.1074/jbc.M117. 792119

Conflict of Interest: The authors declare that the research was conducted in the absence of any commercial or financial relationships that could be construed as a potential conflict of interest.

Copyright $\odot 2021$ Kaushik and Chang. This is an open-access article distributed under the terms of the Creative Commons Attribution License (CC BY). The use, distribution or reproduction in other forums is permitted, provided the original author(s) and the copyright owner(s) are credited and that the original publication in this journal is cited, in accordance with accepted academic practice. No use, distribution or reproduction is permitted which does not comply with these terms. 